\title{
The Furvela tent-trap Mk 1.1 for the collection of outdoor biting mosquitoes
}

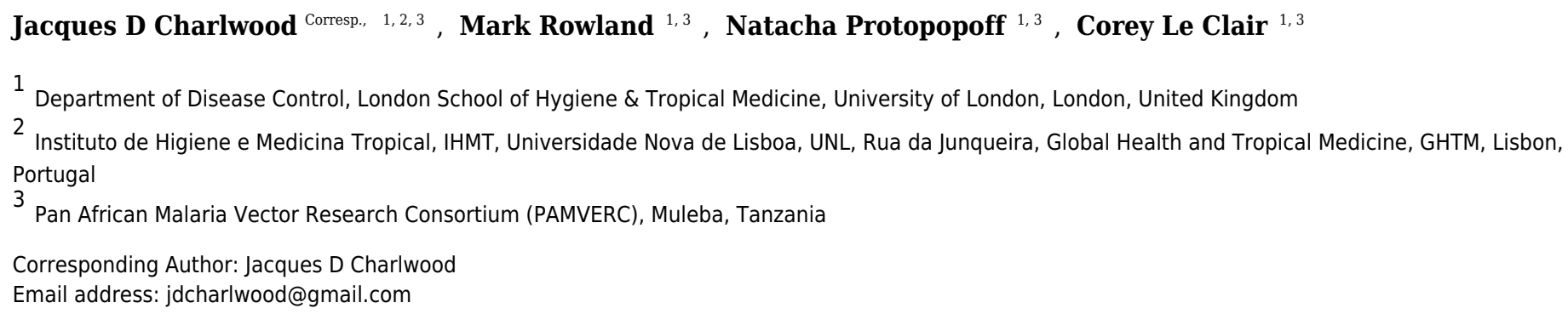

Outdoor transmission of malaria and other vector borne diseases remains a problem. The WHO has recently recognized the need for suitable methods for assessing vector density outdoors and a number of tent-traps have been developed. Only one such trap, the Furvela tent-trap, does not require an 'entry' behavior on the part of the mosquito. It remains the cheapest and lightest tent-trap described. It takes less than two minutes to install and is the only trap that uses readily available components. Here we describe recent modifications to the trap, which make it even easier to set up in the field, provide a standard operating procedure (SOP) and describe some recent experiments examining the effect of the addition of light and door placement to working of the trap. The trap provides the closest approximation to CDC light-traps, widely used to collect indoor biting mosquitoes. This enables the effect of both indoor and outdoor interventions on mosquito density and behavior to be determined. 


\section{The Furvela tent-trap Mk 1.1 for the collection of outdoor biting} 2 mosquitoes

\section{J. Derek Charlwood ${ }^{1,2,3}$ Mark Rowland ${ }^{1,3}$ Natacha Protopopoff 1,3 and Corey LeClair ${ }^{1,3}$}

$7{ }^{1}$ Department of Disease Control, London School of Hygiene and Tropical Medicine, London, UK $8{ }^{2}$ Global Health and Tropical Medicine, GHTM, Instituto de Higiene e Medicina Tropical, IHMT, 9 Universidade Nova de Lisboa, UNL, Rua da Junqueira, Lisbon, Portugal

$10{ }^{3}$ Pan African Malaria Vector Research Consortium (PAMVERC), P.O. BOX 10, Muleba, Tanzania

12 E mail addresses:

13 JD Charlwood - jdcharlwood@gmail.com,

M Rowland - mark.rowland@lshtm.ac.uk

N Protopopoff - nprotopopoff@gmail.com

C Le Clair-corey leclair@hotmail.com,

\section{Abstract}

20 Outdoor transmission of malaria and other vector borne diseases remains a problem. The WHO

21 has recently recognized the need for suitable methods for assessing vector density outdoors

22 and a number of tent-traps have been developed. Only one such trap, the Furvela tent-trap,

23 does not require an 'entry' behaviour on the part of the mosquito and therefore approximates

24 most closely to human landing catches (without the associated risks). It remains the cheapest

25 and lightest tent-trap available, takes less than two minutes to install, and is the only trap that

26 uses readily available components. Here we describe recent modifications to the trap, which 
27 make it even easier to set up in the field, provide a standard operating procedure (SOP), and

28 describe experiments examining the effect of the addition of light and door placement to the

29 working of the trap. In conjunction with CDC light-traps used to collect indoor biting

30 mosquitoes the effect of both indoor and outdoor interventions on mosquito density and

31 behaviour can be determined.

\section{Introduction}

With the current drive to eliminate malaria, worldwide reductions in the disease have occurred

36 (Bhatt et al., 2015), and according to the WHO the incidence of the disease, which takes into

37 account population growth, is estimated to have decreased by $37 \%$ between 2000 and 2015

38 (WHO, 2016).

This success has highlighted the challenges that remain, in particular the control and

40 elimination of residual outdoor transmission not controlled by long lasting insecticide treated

41 nets (LLIN) or Indoor Residual Spraying (IRS) with insecticide. Whilst the monitoring of indoor

42 transmission remains important, outdoor transmission needs to be assessed. Indeed, at the $70^{\text {th }}$

43 World Health Assembly in May 2017, WHO Member States expressed strong support for the

44 strategic approach proposed in the Global Vector Response 2017-2030 which states that:

45 'Assessments of vector populations should use up-to-date methods and techniques to ensure

46 that results are informative for guiding and assessing vector control. Of particular need are

47 robust indicators for vector-borne disease risk, especially in low transmission settings, and

48 methods for assessing vector behaviour such as mosquito outdoor biting.' (WHO, 2016). 
49 Although it is malaria vectors that are of primary concern, many other outdoor biting

50 (exophagic) mosquitoes are potential vectors of pathogens, including a number of 'emerging'

51 diseases.

52

53 The objective of monitoring outdoor biting would be to assess vector species composition,

54 abundance, and the time and place of biting. The primary requisites of such methods are that

55 they catch mosquitoes which would normally bite people outside, using simple and inexpensive

56 equipment. Outdoor exposure, at least in the evening, is best measured in human landing

57 collections ( $\mathrm{HLC}$ ), in which mosquitoes are caught attempting to bite the exposed lower legs of

58 collectors sitting outside, as this is usual behaviour. HLC have been used extensively in the past,

59 largely to sample malaria vectors (Silver, 1998). Landing collections, require considerable

60 supervision however, and, since mosquitoes may be able to inject pathogens before being

61 caught, impose risks to the collectors. Mosquitoes are also attracted to different humans at

62 different rates. Indeed, this differential attraction may also differ by species (Knols et al., 1995).

63

64 In a number of studies commercially available Centre for Disease Control (CDC) light-traps have

65 been used outdoors because they do not expose people to mosquito bites, are widely available

66 and cheap to run (Githeko et al., 1994, Constantini et al., 1998, Cooke et al., 2015). Whilst

67 operationally practical, evidence from these studies suggests that they do not adequately

68 sample the outdoor biting fraction of malaria vectors (Fornadel, Norris and Norris, 2010). Thus,

69 Costantini et al., (1998) reported no significant correlation between Anopheles gambiae s.I.,

70 HLC outdoors and outdoor CDC light-traps, and density-dependent correlation in the case of 
71 Anopheles funestus. Cooke et al. (2015) attempted to measure the outdoor biting fraction of

72 the population by employing a CDC light-trap hung adjacent to an occupied, open-sided rain

73 shelter constructed from a domed one-man tent, but concluded that such traps were a

74 'limitation' of their study. Other traps, notably the MM-X trap (Njiru et al., 2006) and more

75 recently the 'Suna' trap (Homan et al., 2016) have been developed to catch outdoor biting

76 mosquitoes. Nevertheless, they do not provide an easily quantifiable estimate of exposure, nor

77 are they cheap or easily available. Tent-traps are the simplest alternative solution.

DeMeillon (1934) first used tent-traps to collect An. gambiae s.I. in South Africa. He used a plastic gazebo that had openings cut close to the roof that mimicked the eaves in a house.

80 Collectors stood inside the gazebo (their breath and odour attracting mosquitoes) and collected

81 them from the inside walls when the insects were inter-current resting (Mattingly, 1965). Thus,

82 this was more akin to a moveable experimental hut than a trap for collecting outdoor biting

83 mosquitoes.

84 More recently a number of other tent-traps have been developed (Charlwood, 2005, Govella et

85 al., 2009, Krajacich et al. 2014). All but one, however, are similar to the gazebo of DeMeillon in 86 that they require an 'entry' behaviour on the part of the mosquitoes for them to be caught. The

87 usefulness of these tent-traps is limited because not all mosquitoes go inside houses and, as

88 pointed out by Gillies (1974) 'the effectiveness of a baited trap for a particular species of fly

89 primarily depends on its responses to the trapping device in the presence of the attractant

90 stimuli used'.

91 The 'Furvela' tent-trap catches mosquitoes before they enter the tent, it is therefore likely to 92 sample the 'true' outdoor fraction of the population. The trap remains the most 
93 straightforward, lightest and cheapest tent-trap available. It is the only trap that is made from

94 commercially available 'off the shelf' components, including the tent itself. Collections in the

95 Furvela tent-trap are closely correlated to CDC light-trap collections used to monitor indoor

96 biting mosquitoes (Govella et al., 2009, Charlwood et al., 2011, 2012), this makes it especially

97 useful for the measurement of changes in indoor/outdoor ratios following the application of

98 methods to control indoor biting mosquitoes. Since changing the collection bag is easy with the

99 Furvela tent-trap it is also possible to examine the biting profile of the mosquitoes throughout

100 the night as was done previously in Ghana (Charlwood et al., 2011).

101 The trap has been used to make the first map of the spatial variation in outdoor biting densities

102 of mosquitoes (Charlwood et al., 2013) and has recently been used to evaluate an intervention

103 that targeted outdoor biting mosquitoes in Cambodia (Charlwood et al., 2016). Presently they

104 are being used to monitor outdoor biting mosquitoes over a $950 \mathrm{~km}^{2}$ area in 40 villages in 105 Kagera Province, Tanzania.

106 Since its initial description (Charlwood 2005, Govella et al., 2009) the trap has undergone a

107 number of modifications which make it even easier to set up in the field, but do not affect its

108 basic operation. Here we describe these modifications and discuss some recent experiments,

109 including an examination of the effect that the addition of a light to the trap and the effect that

110 door position relative to the sleepers' head has on numbers caught. We supply evidence that

111 the trap does indeed catch outdoor biting mosquitoes and provide a Standard Operating

112 Procedure (SOP) on how to set the trap up in the field.

\section{Methods}


115 The basic principle of the Furvela trap is that host odour and exhaled gases emanating from a

116 gap, the diameter of a CDC trap, in the predominantly closed door of the tent are sucked into a

117 CDC trap (without the light, lid or grid) placed, outside the tent, horizontally between 2 to $3 \mathrm{cms}$

118 from the opening in the door. On approach to the opening the insects are sucked into the trap

119 and held in the standard CDC trap conical collection bag. The suction from the fan effectively

120 prevents any mosquitoes from entering the tent, even at very high densities, so that the sleeper

121 is only exposed if they leave it for some reason. As originally described (Charlwood, 2007,

122 Govella et al., 2013) the setting up of the trap was slightly awkward. Recent improvements to

123 the original trap include the following (Fig 1 A - F):

124 A. The opening is more easily standardised. For this the sides of the tent are sewn back rather than being folded back by clips (although clips can still serve). In addition to standardising the opening, sewing it makes it more difficult for the tent to be zipped up. close to the top of the CDC trap. Short $(6-7 \mathrm{~cm})$ lengths of the same wire are threaded through these holes and medium sized folding-clips attached. The clips are used to attach the trap to the tent.

C. A cover over the collection bag allows collections to continue in the rain. The collection bag has a rain cover (that attaches to the body of the trap with Velcro or a rubber band) sewn over the top half of the net. bag to facilitate attachment. 
137 indoor trapping types with the tent-trap and to investigate possible procedures that might increase the efficiency of the trap. The experiments took place in Kyamyorwa village, located on

154 an inlet of Lake Victoria, in Kagera Province, northern Tanzania before and after an intervention to control malaria was undertaken in the village. The intervention included indoor residual spraying of insecticide for the control of post-prandial insects and the introduction of long-

157 lasting insecticidal nets for the control of biting insects. Indoor walls of houses in the village 
158 were sprayed with pirimiphos-methyl (Actellic) and an LLIN incorporating permethrin and the

159 synergist piperonyl butoxide (PBO) were distributed to residents in February 2015.

160 In addition to CDC light-traps, exit window-traps are another possible proxy for exposure

161 indoors. The number of mosquitoes collected from a $50 \times 50 \times 50 \mathrm{~cm}$, netting sided, window-trap

162 (Silver, 1998) that covered the only window of a bedroom occupied by two adults was

163 compared to the number collected in a tent-trap $20 \mathrm{~m}$ from the house occupied by a single

164 sleeper. The tent-trap was operated twice a week and window-trap collections were

165 undertaken on a daily basis over a nine-week period in Kyamyorwa. Mean numbers per

166 trapping method per ISO week were compared using Pearson's correlation in Excel

167 (supplementary file 2, peerj-16725-Kyamyorwa hse 2 window tent -1.xlsx).

168

169 In order to feed inside a house mosquitoes need to enter through relatively small openings

170 (such as the gap between the eaves and the roof). Not all species of mosquito will do this and

171 so these species are mainly caught biting outdoors. One way of determining if the Furvela tent-

172 trap catches outdoor biting mosquitoes is to determine if these mosquitoes are caught in the

173 trap. We, therefore, compared the species ratios of mosquitoes collected indoors with CDC

174 light-traps or window-traps with outdoor collections in the tent-trap in different situations. In

175 order to determine if the numbers collected varied in the same manner during the night,

176 mosquitoes were removed from the window trap at four-hour intervals $(22: 00,02: 00$ and

177 06:00), whilst at the same time the collection bag on the tent-trap was changed. Collected

178 anophelines were identified (by JDC) using the keys of Gillies and De Meillon (1968) and Gillies

179 and Coetzee (1987). Non-anophelines from Mozambique were kindly identified by $\operatorname{Dr}$ Ralph 
180 Harbach of the British Museum of Natural History, London, those from Tanzania were identified

181 by the authors using the descriptions provided by Gillett (1972).

182

183 As part of the Pan African Malaria Vector Research (PAMVERC) trial (Protopopoff et al., 2017)

184 both tent-traps, outdoors, and light-traps, indoors, are being used to sample malaria vectors in

185 the 48 clusters of the trial area. During each round of sampling, the traps are set up for one

186 night in seven randomly selected houses per cluster. Houses for light-trap and tent-trap

187 samples were chosen at random from the census database obtained at the start of the study.

188 During sampling the light-trap was set up in a bedroom at the end of a bed in which someone

189 slept under a mosquito net and, by one house, a tent-trap was set up (and slept in by the

190 PAMVERC entomologist/collector). Collections from the baseline year (2015) were analysed and

191 are presented. Data were entered into a database and analysed with Stata 12 (Stata, 2013).

192 Since the data were over-dispersed (the deviance was greater than the mean), differences in

193 mosquito density between the two collection methods (light trap or tent trap) were estimated

194 using negative binomial regression. Standard errors were adjusted to allow for within-cluster

195 correlation of responses using robust standard errors.

196

197 Other factors, including number of sleepers, chemical lures, light or the position of the sleepers'

198 head relative to the opening, may affect the efficiency of the trap. Two of these variables were

199 investigated in Kyamyorwa village: the effect of a light source and the effect of door position on

200 the number of mosquitoes captured. The effect of a light source was investigated in a series of 
201 collections using four tent-traps in Kyamyorwa (supplementary file 3, peerj-16725-Light No-

202 Light Tent-Trapping Muleba-1.xls)

203 A new moon occurred on June 272014 (the start of the experiment) and there was little/no

204 ambient illumination during collection dates, providing optimal experimental conditions. We

205 used 2-door tents for these experiments (the Highlander Glen Orchy 2 Tent ${ }^{\circledR}$ ). A standard tent-

206 trap functioned as a control on one side of the tent while the trap on the other door

207 incorporated an incandescent bulb, as used in the CDC light-trap. The trap with the light was

208 rotated between sides on alternate days. Tent-traps were operated from 21:00-06:30 the

209 following day and were operated from June 30-July 4, 2014. The incidence rate ratio (IRR) and

210 density rate ratios (DRR) (i.e. a relative difference measurements used to compare the

211 incidence rates of events occurring at any given point in time) were used to compare the

212 relative density of mosquitoes sampled by a tent-trap with light $(T T+L)$ with a standard trap

213 (TT). Variables including collector, collection date, and sampling site were identified as potential

214 confounding factors during univariate analysis and were included in the final regression model.

216 Since the trap relies on the breath and odour of the host inside the tent, the relative position of

217 the trap to the hosts head might influence the number of mosquitoes collected. We, therefore,

218 determined if the position of the door (at the side or the front) affected numbers collected. The

219 tents used were the two-door Glenn Orchy Highlander (with doors at the sides) and the Taurus

220 Ultra-light two-man tent (with a single door at the front of the tent). Collectors rotated

221 between tents on alternate nights and the tents were rotated every second day. 


\section{Ethics}

224 The collections conducted in Tanzania were done as a component of the Pan African Malaria

225 Vector Research Consortium project 'Evaluation of a novel long lasting insecticidal net and

226 indoor residual spray product, separately and together, against malaria transmitted by

227 pyrethroid resistant mosquitoes' which received ethical clearance from the ethics review

228 committees of the Kilimanjaro Christian Medical College (certificate number 781 on the 16

229 September 2014), the Tanzanian National Institute for Medical Research (20 August 2014), and

230 the London School of Hygiene and Tropical Medicine (reference 6551 on 24 July 2014). The trial

231 was registered with ClinicalTrials.gov (registration number NCT02288637) on 11 July 2014.

232 Collections from Mozambique were undertaken under the aegis of the joint Instituto Nacional

233 de Saúde (INS)-DBL Centre for Health Research and Development project 'Turning houses into

234 traps for mosquitoes', which obtained ethical clearance from the National Bioethics Committee

235 of Mozambique on 2 April 2001 (ref: 056/CNBS/01).

236 Prior to beginning collections, informal sensitisation sessions were conducted with village

237 members to explain sampling-related activities. Written and oral informed consent was

238 obtained from all participants who could withdraw from the study at any time should they wish

239 to do so.

240 Results

241 Indoor outdoor/ratios: Window traps versus Furvela tent-trap

242 In the baseline year, between 23 October 2014 (ISO week number 43) and 14 March 2015 (ISO

243 week number 25), 70 collections were undertaken from the window trap (only two collections

244 being undertaken in December) and 17 tent trap collections were performed. Changes in 
245 population density obtained from the two collection methods were similar $(r=0.93, P<0.001)$

246 (Fig 2). Numbers in the window trap declined from a peak of 1018 on the 24 November 2104 to 247 single figures in the second week of February and from 243 in the tent-trap (on the 19 248 November 2104 to 20 on the 25 February 2015). The decline in both collections fitted a 249 logarithmic series (window $y=-34.45 \ln (x)+120.82 R^{2}=0.793 ;$ tent $y=-37.93 \ln (x)+126.75, R^{2}=$ 250 0.806). The house was sprayed with primiphos-methyl on 23 February 2015 and subsequently 251 the number in the window-trap fell to zero but numbers in the tent-trap persisted, albeit at a 252 very low density. A larger proportion of the catch was caught in the earlier part of the night in 253 the tent-trap compared to the window-trap $\left(X^{2}=16.8, d f=3, P=0.014\right)$ although, for both 254 collections, most insects were caught in the middle hours of the night.

255 Anopheles gambiae was caught in approximately equal numbers from window-trap and tent256 trap whilst other species including Coquelletidia fuscopennata, Mansonia spp. and Culex spp. 257 were collected in greater numbers in the tent-trap compared to the window-trap (Fig 3). 258 Comparable results, between CDC light-trap and Furvela tent-traps, were obtained during the 259 first year of the PAMVERC trial when 34,092 mosquitoes were collected from 3,395 light-trap 260 collections and 495 tent-traps (Table 1).

262 A similar implication comes from the redrawn data from Massavasse in Mozambique, where 263 144,317 mosquitoes were collected from 2,551 light-trap and 94,354 from 776 tent-trap 264 collections (Fig 4) (Charlwood et al., 2013). In Massavasse only An. funestus and Culex spp. 265 (mostly Cx. quinquefasciatus) were caught in greater numbers indoors compared to the other 266 species shown in the figure. Following the application of the insecticide bendiocarb to the 
267 interior walls of houses in the village a greater proportion of the collection of all species,

268 including the exophagic ones, was obtained in tent-traps (Fig 4).

\section{Effect of light on numbers collected}

270 Thirty-two collections (standard tent-trap (TT) $n=16$, and tent-trap+light $(T T+L) n=16$ ) were

271 performed. A total of 180 An. gambiae, 104 Mansonia spp., 195 Cq. fuscopennata and, 140

272 Culex spp. were collected over a 4-day period. Data fit a negative binomial distribution.

273 Surprisingly, the TT+L caught significantly fewer Anopheles females than the TT (Adjusted-

274 IRR=0.56, $P<0.001$ ) (Table 2).

\section{Effects of door position on numbers collected}

276 The rate ratio of the total number of Anopheles captured [IRR 1.05, 95\% $\mathrm{Cl}(0.52-2.12), P=0.9$ ]

277 after 16 collections was not significantly different between the tents, when differences in host

278 attractiveness were taken into account. The IRR of the total number of all mosquitoes captured

279 [IRR 0.92, (95\% C.I. $0.50-1.68), P=0.77]$ was also not significantly different between the tents,

280 when differences in host attractiveness were accounted for. Thus, door position does not

281 affect the efficiency of the trap (Supplementary file peerj-16725-Light No-Light Tent-

282 Trapping Muleba-1.xls).

283

284

285

286

287

\section{Discussion}

In order to determine the density of potential vectors biting outdoors suitable alternative methods to 'gold standard' HLC are required. This is because, in addition to being expensive and requiring considerable supervision, mosquitoes may transmit pathogens before being 
288 caught by collectors performing HLC and so are at risk of becoming ill. The Furvela tent-trap is

289 one such alternative.

290 Like all trapping techniques that rely on the attraction of mosquitoes to a human, including HLC

291 and CDC light-traps, it is likely to be affected by the individual attractiveness of the humans

292 acting as bait. Nevertheless, the greater diversity of species collected in Furvela tent-traps,

293 from a number of study sites, compared to the diversity from indoor collections, especially CDC

294 light-traps, indicates that it adequately samples outdoor biting mosquitoes (Govella et al., 2009,

295 Charlwood et al., 2012). A pairing of either a CDC light-trap or window-trap (used to collect

296 endophagic mosquitoes) with a Furvela tent-trap (to collect exophagic ones) enables the effect

297 of environmental perturbations or interventions to be determined.

298 The data obtained to date with the tent-trap confirm that arbovirus vectors, like Cq.

299 fuscopennata or Mansonia spp., bite predominantly, but not exclusively, outdoors. Among

300 malaria vectors the members of the An. gambiae complex tend to be collected in equal

301 numbers in tent and light-traps whilst An. funestus have generally been caught in light-traps at

302 higher rates compared to tent-traps, confirming their endophilic status. Whether the apparent

303 change from endophilic and endophagic, behaviour to exophagic behaviour in An. funestus

304 following IRS with bendiocarb in Massavasse was because the insects entering houses were

305 killed before being caught in light-traps, or because they refrained from entering in the first

306 place, remains unknown and merits further investigation.

307

308 Mosquito populations, as assessed by indoor collections with CDC light-traps, are often 309 considered to be temporally unpredictable. This may be due to environmental factors, such as 
310 rainfall, that may affect the proportion of the population biting indoors (and so available for

311 capture by the light-trap). For example, in Ghana a greater proportion of the night's catch of

312 An. coluzzii were collected indoors on rainy nights (Charlwood et al., 2011). The total collected

313 in paired indoor light-trap and outdoor tent-trap collections was, however, not different from

314 the number expected. Subsequently the collections returned to the anticipated ratios. In order

315 to control for potential changes in the proportion of the mosquito population biting indoors or

316 outdoors, studies assessing mosquito population dynamics should include simultaneous indoor

317 and outdoor collections. Paired collections may reduce some of the 'noise' in such data making

318 it more amenable to analysis.

319 Vector control plays a big part in current efforts to eliminate malaria. As a result, mosquito

320 densities may become very low. Improving capture efficiency may be useful in such situations.

321 The addition of a light to the trap, however, actually reduced the numbers. This would suggest

322 that the light actually had a repellent effect on the local mosquitoes but why this should be so is

323 not known. Chemical lures may enhance collections and merit consideration.

325 The Furvela tent-trap weighs as little as 2.5 kilograms. Since both CDC light-traps and Furvela

326 tent-traps are portable, effective surveillance, using a limited number of traps, of both indoor

327 and outdoor biting mosquitoes over considerable areas is possible.

328 Mosquito populations can vary as much in space as they do in time (Magbity \& Lines,2002).

329 Determination of high density areas (so called 'hot spots') may enable focussed control, such as

330 targeting selected water bodies for larval control, to be undertaken. In order to determine

331 where high density areas occur maps of mosquito density are required. Ease of transportation 
332 makes the Furvela tent-trap particularly suitable for mapping studies. Mapping using tent-traps

333 enables locations to be determined according the geographical co-ordinates rather than being

334 dependent on where appropriate houses are available for the installation of light-traps. Among

335 mosquitoes that have fixed breeding sites, such as An. funestus, such information may allow

336 estimates of flight range to be obtained, which may also help determine how wide a potential

337 cordon sanitare needs to be for it to be successful (Charlwood et al., 1998).

338

339 A CDC-trap costs 120 US\$ and the cone collection bag 18 US\$ at current prices. A 6V 4.5Ah lead

340 acid rechargeable battery, that costs circa 15 US\$ and weighs $0.7 \mathrm{~kg}$, can power the trap for a

341 night (see the SOP). Two man tents can cost less than $25 \$$. Since the location of the door does

342 not affect collections the choice of tents is large. Simple tents weigh less than 1.8 kilos, hence

343 the total weight of the trap (including the tent) is just over $2 \mathrm{~kg}$ and costs 173 US\$. The

344 professionals who might want to monitor mosquitoes are likely to have CDC light-traps and

345 batteries available. In this case, the trap would cost just 43 US\$. It only requires one person to

346 put it up and, because the interior of the tent is not altered in any way, is comfortable for the

347 sleeper. As can be seen in the video, once the tent itself is up it takes just a few minutes to

348 install. The ease with which collection bags can be changed means that collections can easily be

349 sub-divided throughout the night. As a routine, they can be changed when local residents enter

350 their houses, so that estimates of actual outdoor exposure can be obtained. When not in use

351 the CDC trap can easily be reconverted to a standard light-trap without the need to remove the

352 clips, which hang outside of the trap (see the SOP). 
353 Passive monitoring of mosquito populations is providing information on the distribution of

354 mosquitoes in Europe (Kampen et al., 2015). Presently this is restricted to the collection of 355 insects indoors. The use of the Furvela tent-trap need not be confined to the tropics or to

356 professionals. The very simplicity of the trap means that anyone who goes camping can collect,

357 without risk to themselves, from local outdoor biting fauna. Using a smartphone, collections

358 can be geo-referenced and the locality photographed. Thus, with a minimum amount of

359 professional resources and data collection, national databases (of such things as bird flu

360 vectors) could be established. Data, and eventually samples, might be sent to a central location

361 (such as a Mosquito Abatement Office) where they would be identified and processed. Unlike

362 all other tent-traps the extra equipment required by any sleeper is minimal.

\section{Conclusions}

364

365

Monitoring outdoor biting activity of malaria vectors is an important component of present efforts attempting to control the disease. Our understanding of the ecology of mosquitoes which may be vectors of emerging diseases, other than malaria, is limited. These mosquitoes may well be exophagic. The WHO has recently recognized the necessity for novel sampling tools to conduct surveillance of outdoor biting mosquitoes with the objective of assessing vector species composition, time and place of biting, and abundance (WHO, 2014). Furvela tent-traps

371 are a simple and effective way of collecting such mosquitoes.

\section{Acknowledgements}


374 We would like to thank everyone who has slept in the tents during the studies described here.

375 Special thanks to Elsa Tomás for her help in the design of the original trap and for her ongoing

376 contribution to collections in it. Thanks too, to Judith Cronery for the suggestion to sew open

377 the tent opening. Previously unpublished data form part of the MRC supported PAMVERC

378 project in Muleba, Tanzania. We would also like to acknowledge the improvements to the

379 original manuscript due to the referees and Dominique Shoham for copy editing the

380 manuscript.

\section{References}

382

383

384

385

386

387

388

389

390

391

392

393

394

395

396

397

398

399

400

401

402

403

404
Bhatt, S, Weiss, DJ, Cameron, E, Bisanzio, D, Mappin, B, Dalrymple, U, Battle, KE, Moyes, CL, Henry, A, Eckhoff, PA, Wenger, EA, Briët, O, Penny, MA, Smith, TA, Bennett, A, Yukich, J, Eisele, TP, Griffin, JT, Fergus, CA, Lynch, M, Lindgren, F, Cohen, JM, Murray, CLJ, Smith, DL, Hay, SI, Cibulskis, RE, Gething, PW. 2015. The effect of malaria control on Plasmodium falciparum in Africa between 2000 and 2015. Nature 526(7572):207-211.

Charlwood JD. 2005, A new efficient and cheap trap for the collection of outdoor-biting mosquitoes. Acta Tropica 95:S1-S506.

Charlwood JD, Macia GA, Manhaca M, Sousa B, Cuamba N, Bragança M. 2013. Population dynamics and spatial structure of human-biting mosquitoes, inside and outside of houses, in the Chockwe irrigation scheme, southern Mozambique. Geospatial Health 7(2):309-320.

Charlwood JD, Mendis C, Thompson R, Begtrup K, Cuamba N, Dgedge M, Gamage-Mendis A, Hunt RH, Sinden RE, Høgh B. 1998. Cordon sanitaire or laissez faire: Differential dispersal of young and old females of the malaria vector Anopheles funestus Giles (Diptera: Culicidae) in southern Mozambique. African Entomology 6(1):1-6.

Charlwood JD, Nenhep S, Protopopoff N, Sovannaroth S, Morgan JC, Hemingway J. 2016. Effects of the spatial repellent metofluthrin on landing rates of outdoor biting anophelines in Cambodia, Southeast Asia. Medical and Veterinary Entomology 30(2):229-234. 
405

406

407

408

409

410

411

412

413

414

415

416

417

418

419

420

421

422

423

424

425

426

427

428

429

430

431

432

433

434

435

436

437

438

439

440

441

442

Charlwood JD, Tomás EV, Egyir-Yawson A, Kampango AA, Pitts RJ. 2012. Feeding frequency and survival of Anopheles gambiae in a rice-growing area in Ghana. Medical and Veterinary Entomology 26(3):263-270.

Charlwood JD, Tomás EV, Salgueiro P, Egyir-Yawson A, Pitts RJ, Pinto J. 2011. Studies on the behaviour of peridomestic and endophagic $\mathrm{M}$ form Anopheles gambiae from a rice growing area of Ghana. Bulletin of Entomological Research 101(5):533-539.

Cooke MK, Kahindi SC, Oriango RM, Owaga C, Ayoma E, Mabuka D, Nyangau D, Abel L, Atieno E, Awuor S, Drakeley C, Cox J, Stevenson J. 2015. 'A bite before bed': Exposure to malaria vectors outside the times of net use in the highlands of western Kenya. Malaria Journal, 14:1.

Costantini C, Sagnon NF, Sanogo E, Merzagora L, Coluzzi M. 1998. Relationship to human biting collections and influence of light and bednet in CDC light-trap catches of West African malaria vectors. Bulletin of Entomological Research 88(5):503-511.

De Meillon B. 1935. Entomological studies-Observations on Anopheles funestus and Anopheles gambiae in the Transvaal Johannesburg. Johannesburg: South African Institute for Medical Research 32:195-248.

Fornadel CM, Norris LC, Norris DE. 2010. Centers for Disease Control light traps for monitoring Anopheles arabiensis human biting rates in an area with low vector density and high insecticidetreated bed net use. American Journal of Tropical Medicine and Hygiene 83(4):838-842.

Gillett JD. 1972. Common African Mosquitoes and their Medical Importance. Commonwealth Agricultural Bureau, London.

Gillies MT. 1974. Methods for assessing the density and survival of blood-sucking Diptera. Annual Review of Entomology 19:345-362.

Gillies MT, Coetzee M. 1987. A Supplement to the Anophelinae of Africa South of the Sahara (Afrotropical Region) Publication no. 55. South African Institute for Medical Research, Johannesburg.

Gillies MT, De Mellion B. 1968. The Anophelinae of Africa South of the Sahara (Ethiopian Zoogeographical Region), 2ndPublication no. 54 edn. South African Institute for Medical Research, Johannesburg.

Githeko AK, Service MW, Mbogo CM, Atieli FA, Juma FO. 1994.Sampling Anopheles arabiensis, A. gambiae sensu lato and $A$. funestus (Diptera: Culicidae) with CDC light-traps near a rice 
443 irrigation area and a sugarcane belt in western Kenya. Bulletin of Entomological Research 444 84(3):319-324.

445

446 Govella NJ, Chaki PP, Geissbuhler Y, Kannady K, Okumu F, Charlwood JD, Anderson RA, 447 Killeen GF. 2009. A new tent trap for sampling exophagic and endophagic members of the 448 Anopheles gambiae complex. Malaria Journal 8:1.

449

450 Homan T, Hiscox A, Mweresa CK, Masiga D, Mukabana WR, Oria P, Maire N, Di Pasquale A, 451 Silkey M, Alaii J, Bousema T, Leeuwis C, Smith TA, Takken W. 2016. The effect of mass 452 mosquito trapping on malaria transmission and disease burden (SolarMal): a stepped-wedge 453 cluster-randomised trial. The Lancet, DOI: 10.1016/S0140-6736(16)30445-7

454

Kampen H, Medlock JM, Vaux AGC, Koenraadt CJM, Van Vliet AJH, Bartumeus F, Oltra A, 456 Sousa CA, Chouin S, Werner D. 2015. Approaches to passive mosquito surveillance in the EU. 457 Parasites and Vectors 8:1.

458 Knols BGJ, Takken W, Charlwood JD, De Jong R. 1995. Species-specific attraction of Anopheles mosquitoes (Diptera, Culicidae) to different humans in South-east Tanzania. Proceedings of Experimental and Applied Entomology (N.E.V.) Amsterdam 6: 201-206.

461

Krajacich BJ, Slade JR, Mulligan RT, Labrecque B, Kobylinski KC, Gray M, Kuklinski WS, Burton TA, Seaman JA, Sylla M, Foy BD. 2014. Design and testing of a novel, protective human-baited tent trap for the collection of anthropophilic disease Vectors. Journal of Medical Entomology 51(1):253-263.

Magbity, EB, Lines, JD.2002. Spatial and temporal distribution of Anopheles gambiae s.l. (Diptera: Culicidae) in two Tanzanian villages:

469 implication for designing mosquito sampling routines. Bulletin of Entomological Research 92 (6): 483-488.

471

Mattingly P. 1965. Intercurrent resting, a neglected aspect of mosquito behaviour. Cahiers

Njiru BN, Mukabana WR, Takken W, Knols BGJ. 2006. Trapping of the malaria vector Anopheles gambiae with odour-baited MM-X traps in semi-field conditions in western Kenya. Malaria Journal 5:39. insecticidal net and indoor residual spray product, separately and together, against malaria transmitted by pyrethroid resistant mosquitoes: RCT. The Lancet. 
483

484

485

486

487

488

489

490

491

492

493

494 495

496

497

498

499

Silver JB. 2008. Mosquito Ecology, Field sampling methods. Springer New York. ISBN 978-1-

4020-6665-8

StataCorp. 2013. Stata: Release 12. Statistical Software. College Station, TX: StataCorp LP.

WHO. 2014. Guidance Note on the Control of Residual Malaria Parasite Transmission. Geneva: World Health Organization.

WHO. 2016. World Malaria Report 2016 Geneva: World Health Organization; ISBN: 978924 1511711

WHO. 2017. Global vector control response 2017-2030. Geneva: World Health Organization, 2016. http://www.who.int/malaria/global-vector-control-response/ (accessed May 31, 2017). 
Figure 1 (on next page)

The Furvela tent-trap Mk 1

Modifications and installation of the trap - A The opening of the tent door is sewn open; B Clips are used to attach the body of the trap to the tent; C A rain-proof cover is added to the collection bag; D Eyelets are sewn into the back of the collection bag; $E$ The collection bag is suspended using guy ropes attached to the tent; $\mathrm{F} \mathrm{A}$ footprint that extends under the trap is added. 


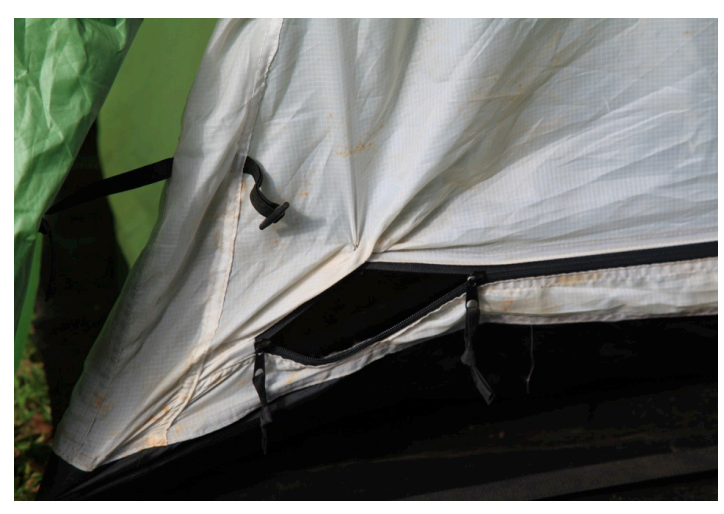

A

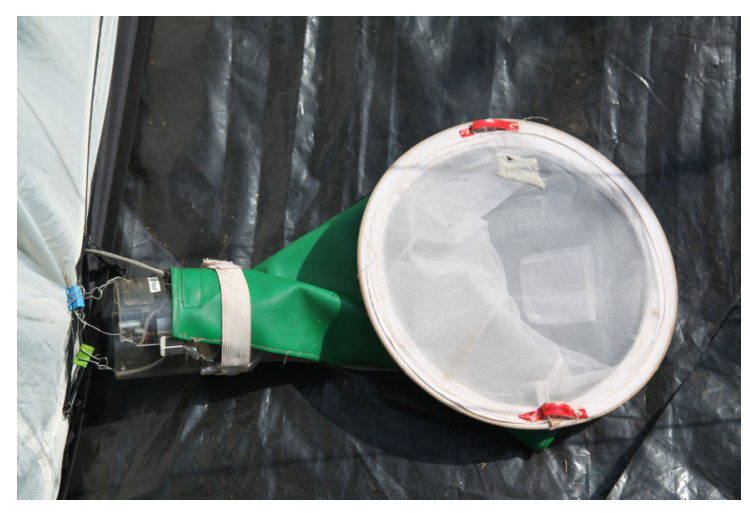

D

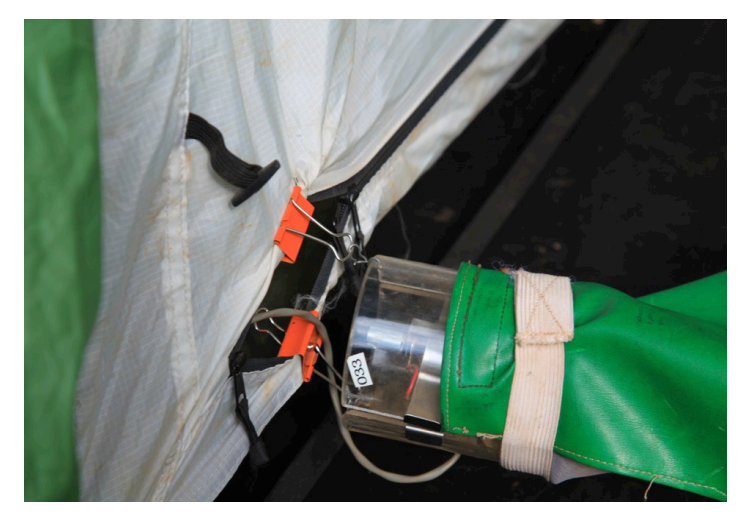

B

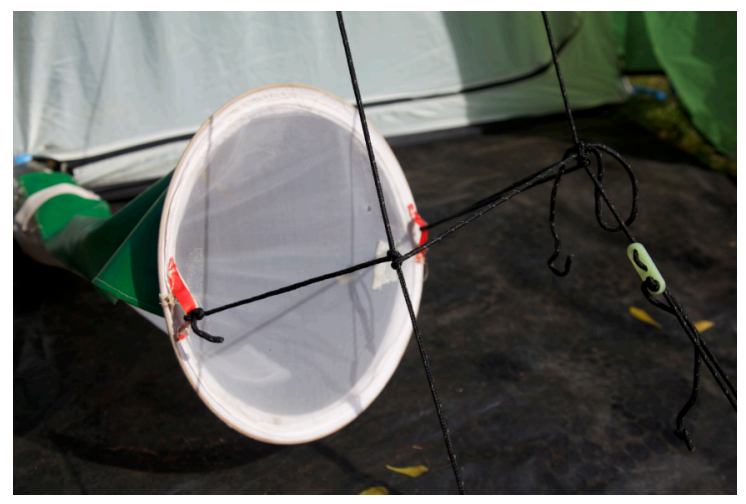

$\mathrm{E}$

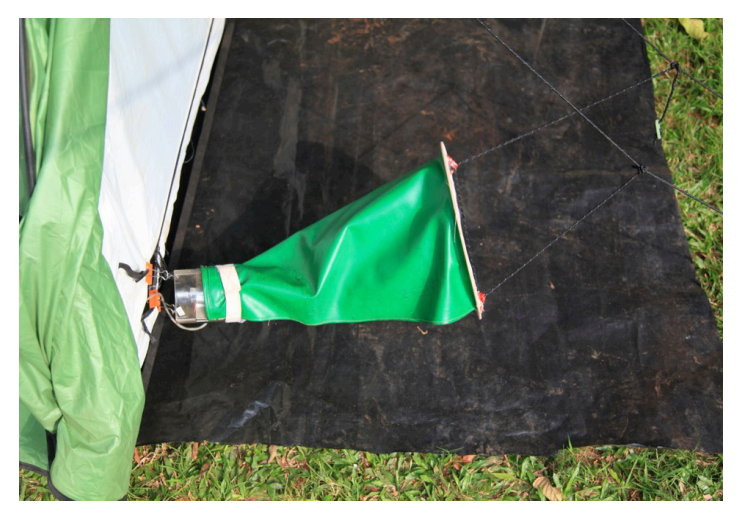

C

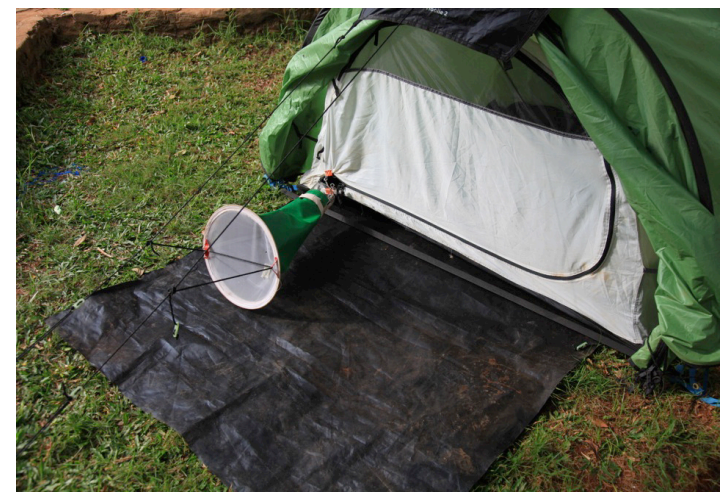

$\mathrm{F}$ 
Figure 2 (on next page)

Exit window trap and Furvela tent- trap collections of An. gambiae s.l. from the village of Kyamyorwa, Muleba District, Kagera Region, Tanznia.

The arrow marks the time when the interior walls of the bedroom were sprayed with pirimiphos-methyl (Actellic) at $1 \mathrm{~g}$ ai per $\mathrm{m}^{2}$ (prior to the spray cross-correlation between mean weekly numbers in the window trap and numbers in the tent-trap $r=0.93, p=$ $>0.001)$. 


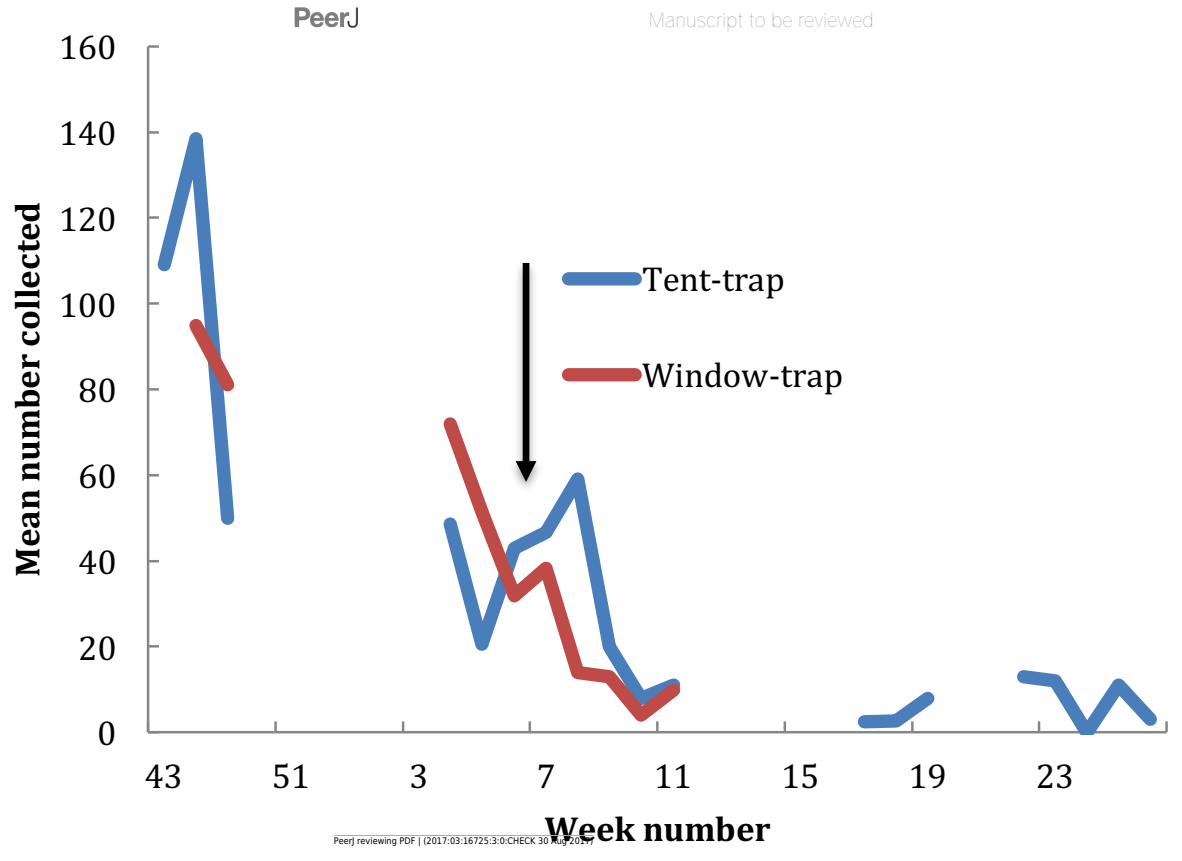


Figure 3 (on next page)

Species comparison between window-traps and Furvela tent-traps

Window-trap tent-trap ratios of mosquitoes from Kyamyorwa, Muleba District, Tanzania A: Anopheles gambiae $(n=10512)$ B: An. funestus $(n=81), C$ : An. coustani $(n=27), D:$ An. zeimanni $(n=282), E: C x$. quinquefasciatus $(n=471), F: C x$. tritaeniorhynchus $(n=21), G$ : Coquelettidia fuscopennata $(n=130), H$ : Mansonia spp. $(n=737)$, : An. squamosus $(n=81)$ and J: An. pharoensis $(\mathrm{n}=49)$. 


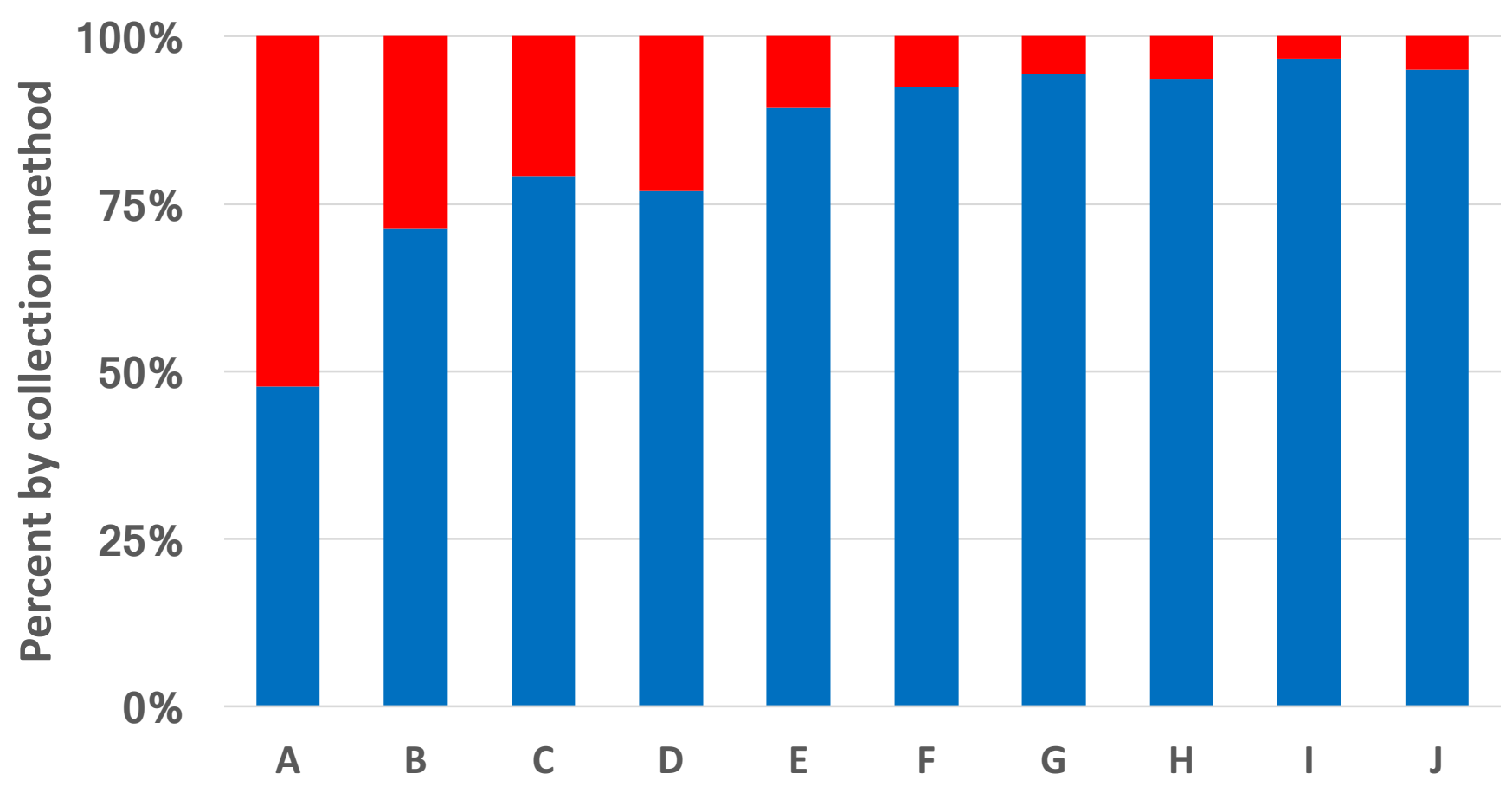




\section{Figure 4 (on next page)}

\section{Indoor and outdoor ratios of mosquitoes from Massavasse}

Indoor and outdoor ratios of the principal mosquitoes collected in Massavasse, Chockwe District, Gaza Province, Mozambique, before (Panel 4A) and after the walls were sprayed with bendiocarb at $0.4 \mathrm{gm}$ ai $\mathrm{m}^{2}$ (Panel 4B). A: Anopheles funestus ( $n$ before spray $=3754, n$ after spray $=1742$ ), B: Culex spp. - mainly $C X$. quinquefasciatus ( $n$ before $=4267, n$ after spray $=2208$ ), C: An. pharoensis ( $n$ before $=2642 n$ after spray $=2078$ ), D: An. arabiensis ( $n$ before $=5406, n$ after spray $=512$ ), E: Aedes scatopagoides ( $n$ before $=1085$ nafter spray $=395)$, F: Mansonia africana ( $n$ before $=38173, n$ after spray $=12054$ ). Data from Charlwood et al., 2013. 


\section{Table $\mathbf{1}$ (on next page)}

Tent-trap and light-trap comparisons from the PAMVERC trial in Muleba District, Tanzania. 


\begin{tabular}{|c|c|c|c|c|c|c|c|c|c|}
\hline & \multicolumn{3}{|c|}{ CDC light-trap } & \multicolumn{3}{|c|}{ Furvela tent-trap } & \multirow[b]{2}{*}{$\begin{array}{c}\text { Density } \\
\text { Ratio }\end{array}$} & \multirow[b]{2}{*}{$95 \% \mathrm{Cl}$} & \multirow[b]{2}{*}{$P$ value } \\
\hline & $\begin{array}{l}\text { Number of } \\
\text { collections }\end{array}$ & Mean & $95 \% \mathrm{Cl}$ & $\begin{array}{l}\text { Number of } \\
\text { collections }\end{array}$ & Mean & $95 \% \mathrm{Cl}$ & & & \\
\hline All vectors & 3,395 & 1.9 & $1.4-2.5$ & 495 & 2 & $1.3-2.6$ & 1 & $0.8-1.2$ & 0.888 \\
\hline All mosquitoes & 3,364 & 8.4 & $5.8-11.0$ & 491 & 11.4 & $7.8-15.0$ & 1.4 & $1.2-1.5$ & $<0.001$ \\
\hline An. gambiae s.l. & 3,395 & 1.7 & $1.2-2.2$ & 495 & 1.8 & $1.2-2.5$ & 1.1 & $0.9-1.4$ & 0.356 \\
\hline An. funestus & 3,395 & 0.3 & $0.2-0.4$ & 495 & 0.14 & $0.07-0.2$ & 0.5 & $0.3-0.7$ & $<0.001$ \\
\hline An. zeimanni & 3,395 & 0.2 & $0.1-0.3$ & 493 & 0.37 & $0.2-0.6$ & 2.1 & $1.0-4.4$ & 0.058 \\
\hline Cx. quinquefasciatus & 3,394 & 3.1 & $1.4-4.8$ & 494 & 3.7 & $1.8-5.5$ & 1.2 & $0.9-1.5$ & 0.197 \\
\hline Mansonia sp. & 3,390 & 1 & $0.7-1.3$ & 495 & 1.5 & $0.9-2.2$ & 1.6 & $1.3-1.9$ & $<0.001$ \\
\hline Cq. fuscopennata & 3,391 & 1.5 & $1.1-1.8$ & 495 & 2.6 & $1.8-3.4$ & 1.8 & $1.3-2.3$ & $<0.001$ \\
\hline
\end{tabular}

1 


\section{Table 2 (on next page)}

Comparison between number of mosquitoes collected between tent-trap and tent-trap with a light source

Comparison between density of species collected in a standard tent-trap ( $T$ ) and tent-trap with an incandescent $6 \mathrm{~V}$ light, as used in a standard CDC light-trap, (TT+ Light), Kyamyorwa, Muleba, Tanzania IRR = Incidence Rate Ratio; Adjusted = IRR adjusted for collector, location, and date; $95 \% \mathrm{Cl}=95 \%$ Confidence Interval 


\begin{tabular}{lllllll}
\hline & \multicolumn{3}{c}{ Anopheles $^{*}$} & Mansonia sp & \multicolumn{2}{c}{ Total All Species } \\
\cline { 2 - 7 } Method & DRR & Adjusted DRR* & DRR & Adjusted DRR* & DRR & Adjusted IRR* \\
\hline Tent-trap & 1.0 & 1.0 & 1.0 & 1.0 & 1.0 & 1.0 \\
Tent-trap+Light & 0.24 & 0.14 & 0.56 & 0.52 & 0.47 & 0.38 \\
95\% CI & $0.08,0.57$ & $0.07,0.28$ & $0.2,1.6$ & $0.27,0.97$ & $0.22,1.02$ & $0.24,0.59$ \\
$p$-value & 0.002 & $<0.001$ & 0.28 & 0.04 & 0.056 & $<0.001$ \\
\hline
\end{tabular}

